\title{
Cigarettes associated with increased mortality in NSTE-ACS
}

A study of patients presenting with non-

ST-segment-elevation acute coronary syndrome (NSTE-ACS) has indicated that cigarette smoking is associated with both the development of coronary artery disease (CAD) and a significantly increased risk of death from this disease. In their study report, the investigators highlight "the importance of continued efforts at smoking cessation and use of appropriate secondary prevention measures in this population".

Cigarette smoking is known to exert prothrombotic effects and, therefore, to be associated with an increased risk of ST-segment-elevation myocardial infarction (STEMI). In line with this known effect of cigarette smoking, smokers are more likely to present with a STEMI than with an NSTE-ACS, a condition that is not effectively treated using thrombolysis.
Notably, among patients who have experienced an acute myocardial infarction and undergone revascularization, smokers have been shown to have lower mortality than nonsmokers. This 'smoker's paradox' is thought to exist because smokers present with acute myocardial infarction at a younger age and with fewer comorbidities than nonsmokers, and because their disease is more likely treatable with thrombolysis.

In the ACUITY population of patients with NSTE-ACS, smokers were 10 years younger than nonsmokers at presentation. Angiographic indices for the extent and complexity of CAD were largely similar in the two patient groups. Univariate analysis indicated that, compared with the nonsmokers in this population, the smokers had $20 \%$ lower risks of 30 -day major bleeding and death within 1 year. However, when age, comorbidities, and other variables were considered in a multivariate analysis, the risk of major bleeding within 30 days was found to be similar for the two patient groups, and the risk of death within 1 year was shown to be $37 \%$ higher for smokers than for nonsmokers. The investigators conclude that their study has shown a "clear and important impact of smoking on mortality and development of CAD in patients with NSTE-ACS”.

Bryony M. Mearns

Original article Robertson, J. 0. et al. Impact of cigarette
smoking on extent of coronary artery disease and
prognosis of patients with non-ST-segment elevation
acute coronary syndromes: an analysis from the ACUITY
(Acute Catheterization and Urgent Intervention Triage
Strategy) trial. JACC Cardiovasc. Interv. doi:10.1016/
j.jcin.2013.11.017

